\title{
High Resolution TEM at 80kV Acceleration Voltage - Is Approaching 1 Ångström Possible?
}

\author{
S. Kujawa, R. Erni, B. Freitag, P. Tiemeijer, M. Stekelenburg \\ FEI Company, Building AAE, P.O. Box 80066, 5600 KA Eindhoven, The Netherlands
}

Beam energy, or equivalently acceleration voltage, should be considered an intrinsic important experimental parameter for high resolution TEM imaging. For each material there is an optimal acceleration voltage that represents the best compromise between sample stability (radiation damage), contrast and resolution. A TEM that allows great flexibility in acceleration voltage operation also allows great flexibility in sample types.

Radiation damage occurs when a specimen is exposed to the TEM electron beam. For organic specimens for example, the primary damage process is the inelastic scattering, which causes molecular excitation or ionization or collective molecular excitation. Since the inelastic crosssection reduces with increasing acceleration voltage, the radiation damage can be reduced by imaging these types of specimen at higher voltages, for example at $300 \mathrm{kV}$ [1].

Next to this damage process, there is a second type of damage. Due to the elastic scattering process, direct displacement of atoms can happen above a so-called energy $(\mathrm{kV})$ threshold [2]. For specimens that are more sensitive to this type of radiation damage process, it is advantageous to reduce the acceleration voltage and find the right compromise between radiation damage and desired lateral resolution and contrast. Carbon Nano Tubes (CNT) are best imaged close to $80 \mathrm{kV}$ for these very reasons.

The Titan 80-300 is optimized for the above described applications and complies with the need for flexibility in acceleration voltage. We investigated the behavior of the Titan operation at $80 \mathrm{kV}$. The investigation was done using a Titan equipped with an image Cs-corrector $[3,4]$. Figure 1 shows a silicon gate oxide image in [110] direction at $80 \mathrm{kV}$. The interface shows that the contrast delocalization effect is dramatically reduced [5] and that it contains a line resolution component down to 1 Ångström! Is Approaching 1 Ångström Possible?

Since the correction of the third order spherical aberration -as achieved by the image correctorreduces the Cs to values typically in $\mu \mathrm{m}$ range, the microscope contrast transfer function (CTF) now depends on the defocus spread caused by the chromatic aberration (temporal coherence) and other incoherent perturbations (e.g. specimen drift). For small defocus, the lateral coherence of the illumination system is negligible. At $80 \mathrm{kV}$, due to the absence of magnetic saturation effects of the objective lens, the chromatic aberration is reduced. Theoretically, this leads to an information limit of about $0.16 \mathrm{~nm}$. In contradiction to this theoretical prediction, we found in our experiments information transfer down to even $0.1 \mathrm{~nm}$. Since at $80 \mathrm{kV}$ the chromatic aberration is dominant factor for resolution limitation, it is expected that the use of a monochromator will even improve the attainable resolution limits. In this contribution we will show new results on this topic. 
The authors thank the employees of CEOS $\mathrm{GmbH}$ for their contributions to making the next generation of corrector technology on Titan a success. The Titan platform is the basis of the TEAM project of the USA Department of Energy with the goal to arrive at 0.5 Ångström resolution in TEM and STEM on a single system, in combination with a Cc corrector developed in co-operation with CEOS GmbH.

[1] R.F.Egerton, Ultramicroscopy 28 (1989) 215

[2] V.E.Cosslett in Electron Microscopy and Analysis 1979 (T.Mulvey, Institute of Physics, London 1979), p. 177

[3] M.Haider et al, Ultramicroscopy 75 (1998) 53

[4] S.Kujawa et al, Microsc. Today 13 no.4 (2005) 16

[5] M.Lentzen et. Al. Ultromicroscopy 92 (2002), 233
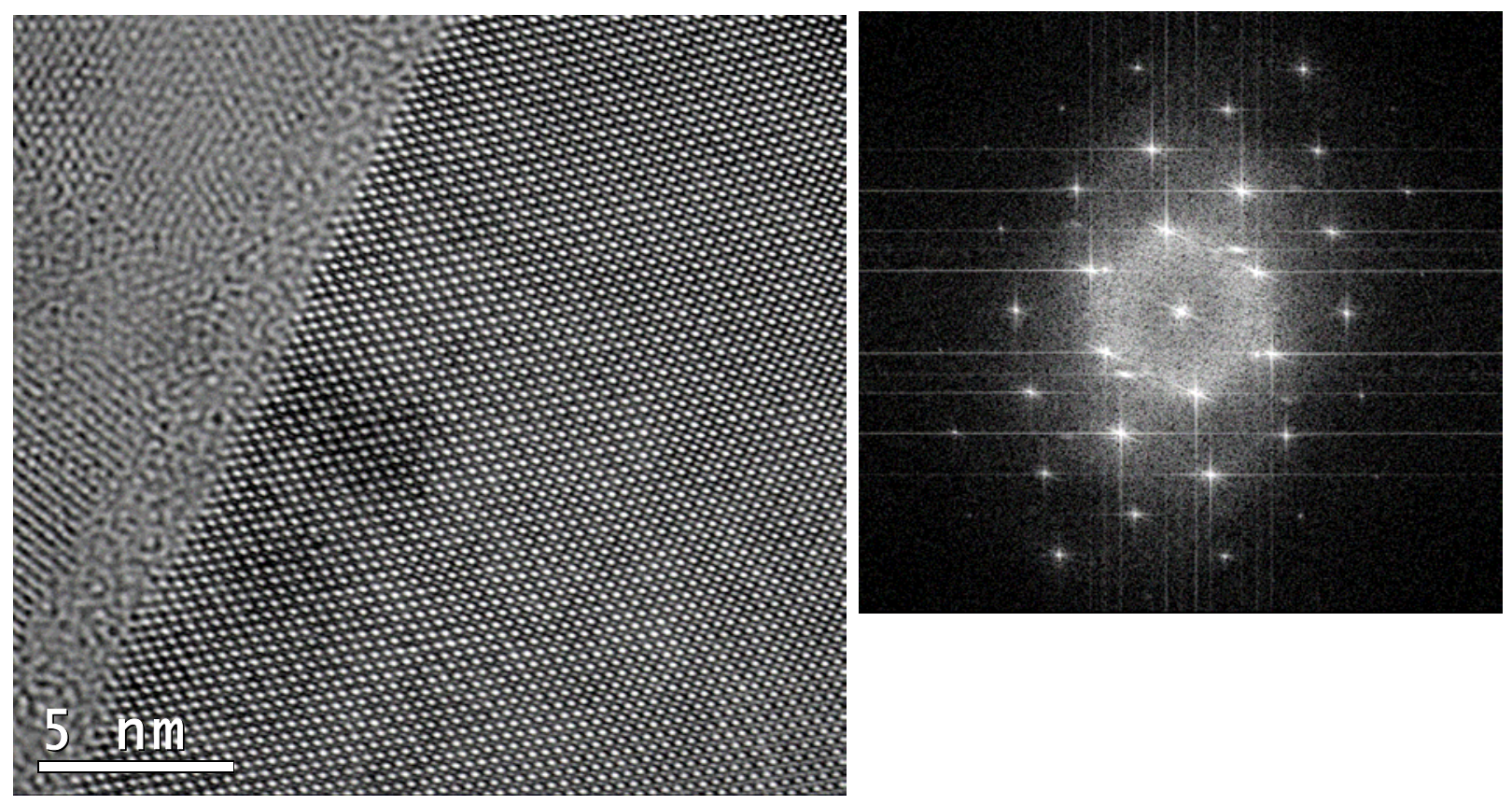

Fig. 1. Gate oxide image in silicon [110] using Titan 80-300 equipped with an image Cs-corrector working at $80 \mathrm{kV}$. The power spectrum shows line resolution down to $0.1 \mathrm{~nm}$. 